\title{
Detection of ISGRI sources in 3 years of BAT all sky survey
}

\section{La Parola, G. Cusumano, A. Segreto, V. Mangano, P. Romano, T. Mineo}

INAF, Istituto di Astrofisica Spaziale e Fisica Cosmica, sez. di Palermo

Via Ugo La Malfa 153, 90146 Palermo, Italy

E-mail: laparoladifc.inaf.it

\section{Ferrigno}

Institut für Astronomie und Astrophysik Tübingen (IAAT)

and

ISDC Data Centre for Astrophysics

Chemin d'Écogia 16, CH-1290 Versoix, Switzerland

\section{G. Chincarini}

Universit'a degli studi di Milano-Bicocca, Dipartimento di Fisica

Piazza delle Scienze 3, I-20126 Milano, Italy

and

INAF, Osservatorio Astronomico di Brera

\section{S. Campana, G. Chincarini, G. Tagliaferri}

INAF, Osservatorio Astronomico di Brera, via E. Bianchi 46

I-23807 Merate, Italy

\section{P. Giommi}

ASI Science Data Center, via Galileo Galilei

I-00044 Frascati, Italy

The Palermo Swift-BAT survey project consists of a systematic search for new hard X-ray sources in the BAT survey data. To this aim, we have developed a dedicated, highly efficient software, the BATIMAGER, completely independent from the one distributed by the BAT hadware team, that processes the data, produces mosaicked sky images and performs source detection and identification. We have applied this code to the first 39 months of survey data, obtaining a list of 968 detections above a signal-to-noise threshod of 4.8 standard deviations. We identify a counterpart for 748 of these sources. Here we present the subsample of the 364 sources that are already known as hard X-ray emitters observed with INTEGRAL-ISGRI or reported in published BAT catalogues and we use them to verify the performance of our software.

7th INTEGRAL Workshop

September 8-11 2008

Copenhagen, Denmark 


\section{Introduction}

The Burst Alert Telescope (BAT [2]) is a coded-mask detector sensitive in the $15-150 \mathrm{keV}$ band, with a field of view of 1.4 steradian (half coded), used as GRB detector on board Swift [5]. While waiting for new GRBs, it continuously collects spectral and imaging information in hard $\mathrm{X}$-rays and accumulates data in "survey" mode covering a fraction between 50 and $80 \%$ of the sky every day. These data provide an almost uniform coverage of the sky (the pointing direction being driven mainly by the random GRB occurrance) and constitute the most extended and sensitive (at least out of the Galactic Plane) survey of the sky in hard X-rays performed so far.

\section{The BATIMAGER}

In order to perform a systematic and efficient search for new hard X-ray sources, we developed the BATIMAGER, a software dedicated to the processing and analysis of the BAT survey data. A complete and detailed description of the BATIMAGER and its performance is presented in [11]. Differently from the software developed by the BAT hardware team [8], where the final products are derived through the application of a number of different procedures in a pipeline, our software integrates all processing steps (from shadowgram accumulation to mosaicking) in one single program, that accepts a list of $\mathrm{DPH}^{1}$ files as input, and returns the final mosaic and all the relevant products as output. This has the obvious advantage of a large saving in terms of CPU load and (without the need of saving a large amount of intermediate products) disk memory and I/O time. The code also provides some tools for the in-flight calibration of the telescope: equalization of the detector pixel array, modelling of the coded mask pattern, boresight correction.

The main steps performed by the BATIMAGER are: cross correlation of each shadowgram with the mask pattern (balanced cross-correlation performed through FFT), detection and subtraction of bright sources, background modelling and subtraction, mosaicking, source detection on the final mosaic. The source identification is performed using a catalogue that includes all the sources detected with INTEGRAL-ISGRI ${ }^{2}$ and the sources already reported by other authors [9, 13, 1] as detected with BAT.

\section{Known sources in the BAT survey}

We applied the BAtImager to the first 39 months of Swift-BAT survey data. A complete description of this analysis and the resulting catalogue of 748 identified sources is reported in [3]. 324 of the identified sources are already known X-ray emitters, as they have been already detected with INTEGRAL-ISGRI; 40 more sources have been already reported by [9, 13, 1]. We used this sample of 364 sources (or part of it, if more appropriate) as a calibrator set for the boresight correction and the error circle evaluation, and to test the overall performance of the BATIMAGER. 
Error radius (95\%): 1.47 arcmin

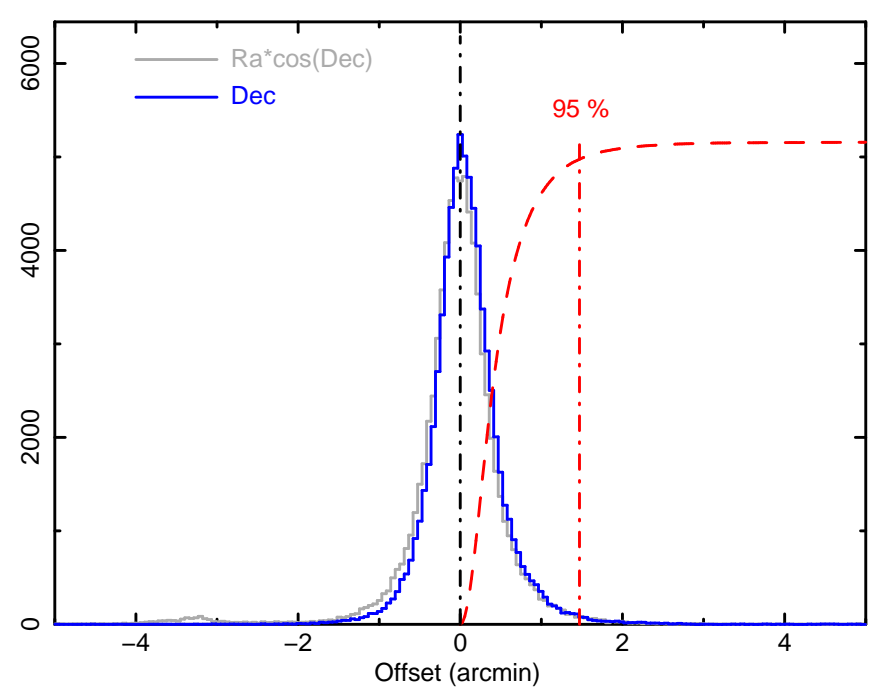

Figure 1: Distributions of the $\mathrm{Ra} \times \cos (\mathrm{Dec})$ (grey) and Dec (blue) offsets between the measured and the nominal position of the bright sources detected in single pointing reconstucted images after the boresight correction. The red dashed line is the cumulative offset distribution.

\section{Boresight correction}

Any misalignment between the satellite star-trackers and the reference system of the BAT instrument produces a degradation of the spatial resolution (boresight error). In order to correct for this effect we use the position obtained with the BATIMAGER for bright identified sources detected in single DPHs. This data set is quite uniformly distributed over the BAT field of view. We evaluated the offset between the detected position and the nominal position of these sources. The resulting offset distribution shows both a radial and an azimuthal dependence and has been used to derive the parameters that minimize the discrepancy between the measured and nominal positions over the entire field of view. The correction has required the evaluation of the following parameters: three parameters determining the rotation angles between the star-trakers and the BAT references system; two parameters determining the translation between the coded mask and the detector plane; one amplification factor depending on the distance between the coded mask and the detector plane and on the size of the detector plane. This boresight correction is time dependent because the data accumulated between 2007 July 27 and 2007 September 11 required four different boresight solutions to account for the final change in the star-tracker configuration.

Fig. 1 1 shows that the $\operatorname{Ra} \times \cos (\mathrm{Dec})$ and Dec offsets distributions after the boresight correction have a Gaussian shape with a standard deviation of about 0.37 arcmin and that the $95 \%$ containment radius of all the positions of bright sources measured over the entire FOV is $1.47 \mathrm{arcmin}$.

\footnotetext{
${ }^{1}$ The BAT survey data are stored in Detector Plane Histograms (DPHs) i.e. three-dimensional arrays (two spatial dimensions, one spectral dimension) that contain an image of the detector plane for each spectral channel

${ }^{2}$ http://isdc.unige.ch/Data/cat/latest/catalog.html
} 


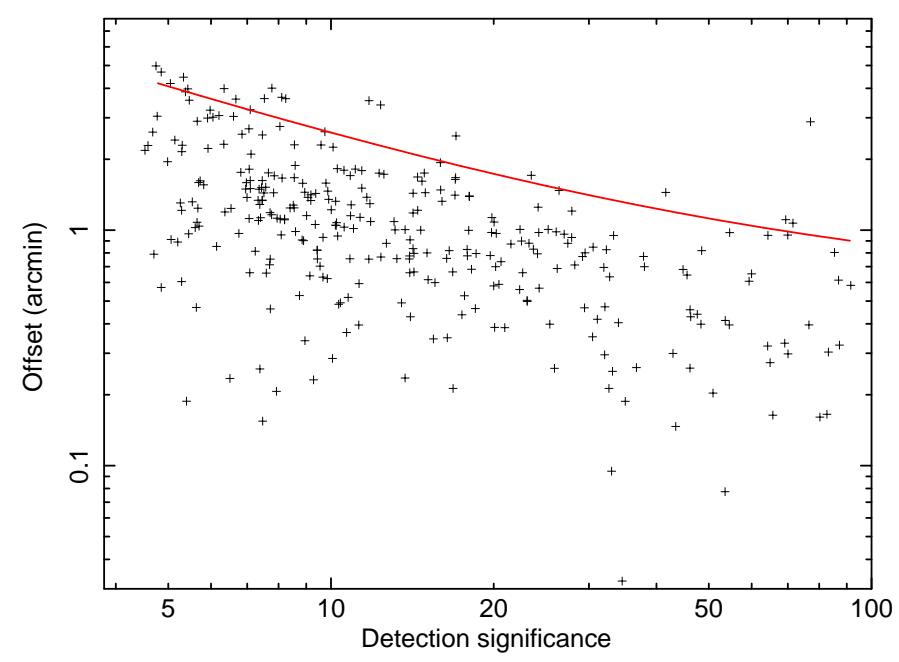

Figure 2: Distribution of the offsets between the position measured in the BAT mosaic sky maps and that of the catalogued counterpart as a function of the detection significance for the 364 known X-ray emitters detected with BatImager in the BAT survey data. The red line is $95 \%$ error radius.

\section{Position accuracy evaluation}

It can be shown that the average point source location statistical error for an optimum coded aperture system with a defined PSF scales inversely with the source detection significance, defined as the ratio between the reconstructed source peak and the computed statistical standard deviation associated to the peak pixel (see e.g. [ஞ). However, in a real instrument, the systematic errors on the reconstructed image limit the localization accuracy above a constant plateaux.

The position accuracy achieved with the BATIMAGER was evaluated in the following steps: - we divided the 39 month data set in 20 time intervals and produced a mosaic for each of them; the detection lists were cross-correlated with the ISGRI catalogue extended with the BAT sources reported in [9, 13, 1];

- we derived the offsets between the coordinates of the sources identified in each mosaic and the nominal counterpart coordinates;

- in order to evaluate the position accuracy dependence on the source significance, we extracted the $95 \%$ radius containment for different significance intervals;

- we fit the radius containment values vs. the detection significance with a power-law plus a constant, obtaining the following best fit equation:

$$
R_{95}=12.5 \times S^{-0.78}+0.54
$$

where $R_{95}$ is the $95 \%$ error radius (in arcmin) for the source position and $S$ is the detection significance measured on the sky mosaic. We verified our result comparing this expression with the offset distribution of the sources detected and identified in the all-sky mosaic of the whole 39 months data set. Fig. 国 shows the $95 \%$ error radius (red line) and the offset distribution. 

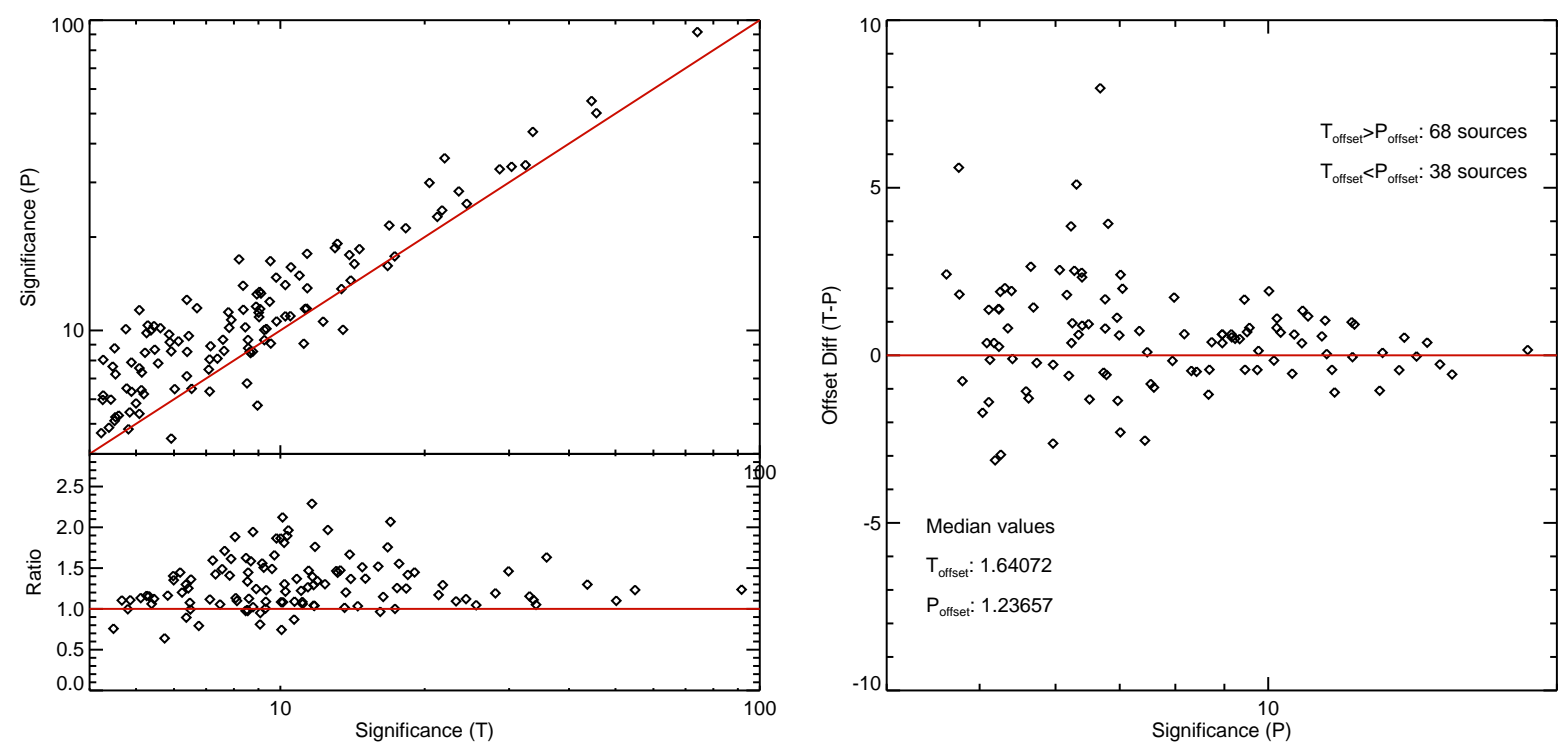

Figure 3: Left panel: Comparison between the source significance ( $\mathrm{Y}$ axis) reported in [13] $(\mathrm{T})$ and the detection significance obtained for the same sources with BATIMAGER on the same set of survey data (P). Right panel: Comparison between the offset of the BAT sources with respect to the identified counterpart. The $\mathrm{Y}$ axis represents the difference between the offset in [13] (Baumgartner, private communication) and the offsets of the same sources obtained from the analysis of the same dataset with our code.

\section{The BatImager performance}

In order to verify the performance of the BATIMAGER, we compared the results reported in [13], obtained using the Swift-BAT team software, with the results of our code on the same survey data set (9 months starting mid-December 2004). Figure 3 (left panel) compares the significance reported in [13] Table 1 (defined as in [9]) and the significance defined in the same way we obtain with the BATIMAGER for the same sources (see details in [11]). We find that with the BATIMAGER we achieve larger significance for more than $90 \%$ of the sample. On average, the improvement in significance is of $\sim 30 \%$. In Figure 3 (right panel) we compare the offset of the BAT sources with respect to the identified counterpart: we note that the BATIMAGER yields a better location accuracy than the Swift-BAT team software for $64 \%$ of the sources. The median value of the offset distribution for the sources in [13] (Baumgartner, private communication) is 1.64 arcmin, while the median value for the sources detected with BATIMAGER is 1.24 (a 25\% improvement in localization accuracy).

\section{Summary}

We report the first results of the Palermo BAT survey project, showing how our newly developed software, the BATIMAGER, allows for an optimal exploitation of the BAT data, and how the known hard X-ray sources we detect in the first 39 months of survey data have been used to calibrate the software. More details on the method and results of the project can be found in these proceeding also in [12, 4, 7, 10]. 


\section{Aknowledgement}

This research has made use of NASA's Astrophysics Data System Bibliographic Services, of the SIMBAD database, operated at CDS, Strasbourg, France, as well as of the NASA/IPAC Extragalactic Database (NED), which is operated by the Jet Propulsion Laboratory, California Institute of Technology, under contract with the National Aeronautics and Space Administration. This work was supported by MIUR grant 2005-025417 and contract PRIN INAF CRA 1.06.10.07.

Italian researchers acknowledge the support of Nature $(455,835-836)$ and thank the Editors for increasing the international awareness of the current critical situation of the Italian Research.

\section{References}

[1] Ajello, M., Greiner, J., Kanbach, G., Rau, A., Strong, A. W., \& Kennea, J. A. 2008, BAT X-Ray Survey. I. Methodology and X-Ray Identification, ApJ, 678, 102

[2] Barthelmy, S. D., et al. The Burst Alert Telescope (BAT) on the SWIFT Midex Mission, Space Science Reviews, 2005, 120, 143

[3] Cusumano G., La Parola V., Segreto A., Mangano V., Ferrigno1 C., Romano P., Mineo T., Campana S., Chincarini G., Giommi P., Moretti A., Tagliaferri G., The Palermo Swift-BAT hard X-ray catalogue.II. Results after 39 months of sky survey 2009, A\&A, submitted

[4] Cusumano G. et al., New hard X-ray emitters discovered by BAT, 2009, in proceeding of 7th INTEGRAL Workshop, BoS (Integra108) 134

[5] Gehrels, N., et al., The Swift Gamma-Ray Burst Mission, 2004, ApJ, 611, 1005

[6] Gros, A., Goldwurm, A., Cadolle-Bel, M., Goldoni, P., Rodriguez, J., Foschini, L., Del Santo, M., Blay, P., The INTEGRAL IBIS/ISGRI System Point Spread Function and Source Location Accuracy, 2003, A\&A, 411, L179

[7] Mangano, V. et al. Hard X-ray search for unidentified EGRET sources in the BAT and ISGRI survey archives, 2009, in proceeding of 7th INTEGRAL Workshop, PoS (Integral08) 133

[8] Markwardt et al. 2007, The SWIFT BAT Software Guide (Version 6.3), http://swift.gsfc.nasa.gov/docs/swift/analysis/bat_swguide_v6_3.pdf\}

[9] Markwardt, C. B., Tueller, J., Skinner, G. K., Gehrels, N., Barthelmy, S. D., \& Mushotzky, R. F., The Swift/BAT High-Latitude Survey: First Results, 2005, ApJL, 633, L77

[10] Sbarufatti, B. et al., Search for Roma-BZCAT Blazars in the Palermo BAT Survey archive 2009, in proceeding of 7th INTEGRAL Workshop, POS (Integral08) 069

[11] Segreto A., Cusumano G., Ferrigno C., La Parola V., Mangano V., Mineo T., Romano P., The Palermo Swift-BAT Survey. I. Methodology, 2009, A\&A, submitted

[12] Segreto, A. et al. Hard X-ray surveys with coded mask telescopes, 2009, in proceeding of 7th INTEGRAL Workshop, BoS (Integra108) 131

[13] Tueller, J., Mushotzky, R. F., Barthelmy, S., Cannizzo, J. K., Gehrels, N., Markwardt, C. B., Skinner, G. K., \& Winter, L. M., Swift BAT Survey of AGNs, 2008, ApJ, 681, 113 